\title{
Texture Alignment in Simple Shear
}

\author{
Frédéric Dufour $^{1}$, Hans Mühlhaus ${ }^{2}$, and Louis Moresi ${ }^{3}$ \\ 1 Laboratoire de Génie Civil de Nantes - Saint-Nazaire \\ 1 rue de la Noë, 44321 Nantes Cedex, France \\ frederic.dufour@ec-nantes.fr \\ ${ }^{2}$ Queensland University, Advanced Centre for Earthquakes Studies \\ St Lucia, QLD 4072, Australia \\ 3 MONASH University \\ PO Box 28M, Vic 3800, Australia
}

\begin{abstract}
We illustrate the flow behaviour of fluids with isotropic and anisotropic microstructure (internal length, layering with bending stiffness) by means of numerical simulations of silo discharge and flow alignment in simple shear. The Cosserat theory is used to provide an internal length in the constitutive model through bending stiffness to describe isotropic microstructure and this theory is coupled to a director theory to add specific orientation of grains to describe anisotropic microstructure. The numerical solution is based on an implicit form of the Material Point Method developed by Moresi et al. [1].
\end{abstract}

Keywords: director theory, Cosserat theory, grain flow, Lagrangian integration points

\section{Introduction}

The mechanics of granular materials has intrigued physicists and engineers for well over two centuries. At low strain rates, particulates such as sand or cereals behave like solids, but at high strain rates, the behaviour is fluid or gas like.

Computer simulations and specifically discrete element simulations 2] are an important tool for exploring the fundamental behaviours of granular flows, flow regimes, phase transitions, fluctuations etc. However computational requirements set strong limitations to the size of discrete element models. The much simpler and (where applicable) much more efficient continuum models for granular flows are valid when the typical grain size is much smaller than characteristic structural dimensions e.g. the outlet size in silo flows. Cosserat continuum theory [3] considers some of the salient features of the discrete microstructure (e.g. grain size, relative rotation between microstructure and the continuum) within the framework of a continuum theory. Such a theory fits between detailed discrete theories and the usual continuum theory.

The Cosserat- or micropolar theory may be employed for a variety of applications involving the need to describe the heterogenous microstructure of the material such as granular materials [4], layered materials [5] or crystals [6] within the framework of a continuum theory. 
This class of theories can be implemented in the context of a classical finite element method (FEM). However, very large deformations are sometimes difficult to handle elegantly within the FEM because mesh distortion and remeshing can quickly present severe difficulties. The version of the Material Point Method (MPM, [7] and [8]) applied here combines the versatility of the standard FEM with the geometrical flexibility of pure particle schemes such as the Smooth Particle Hydrodynanmics (SPH, [9]).

In MPM Lagrangian integration points move through a spatially fixed Eulerian mesh. MPM is inspired by particle-in-cell (PIC) finite difference methods, originally designed for fluid mechanics, in which fluid velocities are solved on the mesh, and material strains and material history variables are recorded by Lagrangian particles. These particles serve as integration points for the stiffness matrix and force vectors. The MPM method applied here is implicit [1] as opposed to the explicit form proposed by Sulsky et al. [10].

We show simple simulations to illustrate the performance of the numerical scheme and the constitutive theory.

\section{Mathematical Formulation}

\subsection{Cosserat Deformation Measures}

We assign a local rigid cross to every material point $\left(x_{1}, x_{2}, x_{3}\right)$ of the body in a Cartesian coordinate system $\left(X_{1}, X_{2}, X_{3}\right)$. During deformation, the rigid crosses rotate at a rate $\omega_{i}^{c}$ about their axis $i$ and are translated according to the conventional linear velocity vector $\mathbf{u}$. The angular velocity $\omega_{i}^{c}$ is considered to be independent of $\mathbf{u}$ and differs from the angular velocity of an infinitesimal volume element of the continuum $\omega_{i}$,

$$
\omega_{i}=-\frac{1}{2} \epsilon_{i j k} W_{j k}
$$

where

$$
W_{j k}=\frac{1}{2}\left(u_{j, k}-u_{k, j}\right)
$$

In (1) $\epsilon_{i j k}$ designates the permutation symbol, and in (2) $(.)_{, k} \equiv \partial(.) / \partial x_{k}$ are partial derivatives.

In the classical theory the stretching tensor is given by:

$$
D_{i j}=\frac{1}{2}\left(u_{i, j}+u_{j, i}\right)
$$

In the Cosserat theory, as a rotational parameter has been added, in addition to the classical strain rate tensor $\boldsymbol{D}$, there is an additional rate measure,

$$
\omega_{i}^{r e l}=\omega_{i}-\omega_{i}^{c}
$$

which represents the relative angular velocity between the material element and the associated rigid coordinate cross. In this case, the rate of the deformation 
tensor can be expressed by the rate of the distortion tensor, $\gamma$,

$$
\gamma_{i j}=u_{i, j}-W_{i j}^{c}
$$

where

$$
W_{i j}^{c}=-\epsilon_{k i j} \omega_{k}^{c}
$$

and by the tensor representing the measure of relative angular velocity between the neighbouring rigid crosses,

$$
\kappa_{i j}=\omega_{i, j}^{c}
$$

The conventional strain rate tensor can be expressed as the symmetrical part of the rate of the distortion tensor,

$$
D_{i j}=\frac{1}{2}\left(\gamma_{i j}+\gamma_{j i}\right)
$$

and the relative angular velocity as the antisymmetrical part

$$
W_{i j}^{r e l}=\frac{1}{2}\left(\gamma_{i j}-\gamma_{j i}\right)
$$

We have 2 deformation rate measures i.e. $\gamma$ and $\kappa$. Both measures are objective. In a rotating observer frame $\gamma$ and $\kappa$ are obtained as $Q \gamma Q^{T}$ and $Q \kappa Q^{T}$ where $Q Q^{T}=1$ describes the rotation of the moving -with respect to the fixed-observer frame.

\subsection{Constitutive Relationships for Granular Materials}

In a $2 \mathrm{D}$ conventional continuum an isotropic material is characterised by a bulk viscosity $B$ and a shear viscosity $\eta$, for a Cosserat continuum we also have a Cosserat shear viscosity $\eta^{c}$ and a bending viscosity $M$. The constitutive relation for a generalised Newtonian fluid can be written in the usual pseudo-vector form:

$$
\sigma=\Lambda D
$$

where the stress vector components are:

$$
\boldsymbol{\sigma}^{T}=\left\{\sigma_{x x}, \sigma_{y y}, \sigma_{x y}, \sigma_{y x}, \mu_{z x}, \mu_{z y}\right\}
$$

the deformation vector components are:

$$
D^{T}=\left\{\gamma_{x x}, \gamma_{y y}, \gamma_{x y}, \gamma_{y x}, \kappa_{z x}, \kappa_{z y}\right\}
$$

and the matrix $\boldsymbol{\Lambda}$ is expressed as:

$$
\left\{\begin{array}{cccccc}
B+\eta & B-\eta & 0 & 0 & 0 & 0 \\
& B+\eta & 0 & 0 & 0 & 0 \\
& & \eta+\eta^{c} & \eta-\eta^{c} & 0 & 0 \\
& & & \eta+\eta^{c} & 0 & 0 \\
& \text { symm. } & & & M & 0 \\
& & & & & M
\end{array}\right\}
$$


For benchmarking purposes, we use the simplest possible realisation of a granular, viscous medium. In the granular-elasticity model of Choi and Mühlhaus [1] we replace the contact stiffnesses $K_{n}$ and $K_{m}$ and relative displacements by contact dashpots $\eta_{n}$ and $\eta_{m}$ and relative velocities and relative rotation rates and obtain the relationships

$$
\begin{aligned}
\eta & =\frac{1-n}{4 \pi} k\left(\eta_{n}+\eta_{m}\right) \\
\eta^{c} & =\frac{1-n}{2 \pi} k \eta_{m} \\
B & =\frac{1-n}{2 \pi} k \eta_{n} \\
M & =2 \eta^{c} R^{2}
\end{aligned}
$$

\subsection{Director Theory}

The salient mechanical feature of layered geomaterials is the existence of a characteristic orientation given by the normal vector field $n_{i}\left(x_{k}, t\right)$ of the layer planes, where $\left(x_{1}, x_{2}, x_{3}\right)$ are Cartesian coordinates, and $t$ is the time. We assume linear viscous behaviour and designate with $\eta$ the normal viscosity and $\eta_{s}$ the shear viscosity in the layer planes normal to $n_{i}$. The orientation of the normal vector, or director as it is sometimes called in the literature on oriented materials, changes with deformation. Using a standard result of continuum mechanics, the evolution of the director of the layers is described by

$$
\begin{gathered}
\dot{n}_{i}=W_{i j}^{n} n_{j} \text { where } W_{i j}^{n}=W_{i j}-\left(D_{k i} \lambda_{k j}-D_{k j} \lambda_{k i}\right) \\
\text { and } \lambda_{i j}=n_{i} n_{j}
\end{gathered}
$$

where $\boldsymbol{L}=\boldsymbol{D}+\boldsymbol{W}$ is the velocity gradient, $\boldsymbol{D}$ is the stretching and $\boldsymbol{W}$ is the spin. The superscript $n$ distinguishes the spin $\boldsymbol{W}^{n}$ of the director $\boldsymbol{N}$ from the spin $\boldsymbol{W}$ of an infinitesimal volume element $d V$ of the continuum.

\subsection{Specific Viscous Relationships}

We consider layered materials. The layering may be in the form of an alternating sequence of hard and soft materials or in the form of a superposition of layers of equal width of one and the same material, which are weakly bonded along the interface. In the following simple model for a layered viscous material we correct the isotropic part $2 \eta D_{i j}^{\prime}$ of the model by means of the tensor $\boldsymbol{\Lambda}$ to consider the mechanical effect of the layering; thus

$$
\sigma_{i j}=2 \eta D_{i j}^{\prime}-2\left(\eta-\eta_{s}\right) \Lambda_{i j l m} D_{l m}^{\prime}-p \delta_{i j}
$$

where $p$ is the pressure, $\boldsymbol{D}^{\prime}$ designates the deviator of $\boldsymbol{D}\left(\right.$ i.e. $\boldsymbol{D}^{\prime}=\boldsymbol{D}-\operatorname{tr}(\boldsymbol{D})$ ), and

$$
\Lambda_{i j k l}=\left(\frac{1}{2}\left(n_{i} n_{k} \delta_{l j}+n_{j} n_{k} \delta_{i l}+n_{i} n_{l} \delta_{k j}+n_{j} n_{l} \delta_{i k}\right)-2 n_{i} n_{j} n_{k} n_{l}\right)
$$




\section{Applications}

\subsection{Silo Discharge}

We now consider the discharge of a Cosserat material from a model silo. In this case we are interested in the influence of the internal length parameter on the discharge velocity of a Cosserat fluid as described by (10 13)

Figure 1 shows the geometry of the model as well as different snapshots along the computations. Heavy lines are free-slip boundaries. Flow is only due to the downward gravity field. The grid drawn on the flowing material is a "dye" to record deformation - it does not affect the material properties. Corridors along the edges provide space where upward flow of the passive background material can take place to equilibrate the pressure due to the downward flow of the Cosserat viscous material. The mechanical characteristics of each material are summarized in Fig. 2.

In Fig. 3 we plot the volume flowing out the reservoir versus time and for different values of the ratio $\alpha=R / a$ where $a$ is the silo aperture. The flow rate is almost identical for all values of $\alpha$ (Fig. 3 and 3 e) larger than the aperture (1.0) which corresponds, in the elastic case, to a situation in which no flow can occur. Note that in purely viscous materials static equilibrium states do not exist, ensuring that stable arches do not form. For $0 \leq \alpha \leq 1.0$, the smaller the internal length the faster the outflow. As for the elastic case the internal length provides a bending stiffness which slows down the flow.

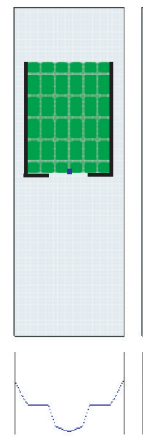

(a)
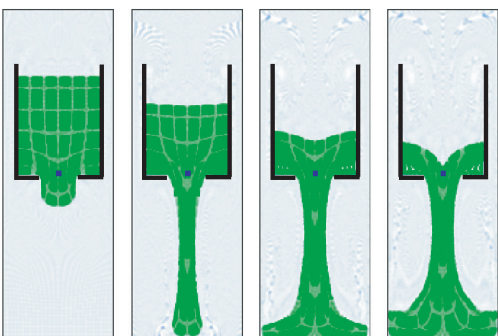

(b)

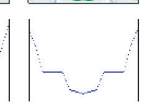

(c)

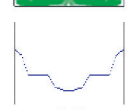

(d)

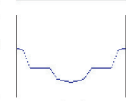

(e)

Fig. 1. Snapshots of the model. (a) Initial conditions, (b) $t=4.7510^{-4} \mathrm{sec}$, (c) $t=1.4210^{-3}$ sec., (d) $t=2.8510^{-3}$ sec. and (e) $t=3.810^{-3}$ sec.

\begin{tabular}{|c|c|c|}
\hline & Granular material & Background \\
\hline Internal length & $R$ & 0 \\
\hline Shear viscosity & 1000 & 1 \\
\hline Bulk viscosity & $+\infty$ & $+\infty$ \\
\hline Density & $10^{6}$ & 0 \\
\hline
\end{tabular}

Fig. 2. Constant values. 


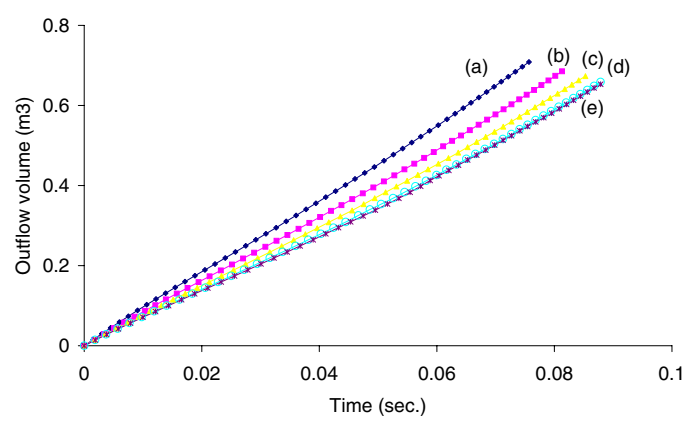

Fig. 3. Outgoing volume for (a) $\alpha=0$, (b) $\alpha=1 / 3$, (c) $\alpha=2 / 3$, (d) $\alpha=5 / 3$ and (e) $\alpha=10 / 3$

\subsection{Self-Alignment}

In this example the flow of anisotropic particles (pencil-shaped) is modelled in an infinite shearlayer. The normal viscosity $\eta$ is set to $1000 \mathrm{~Pa}$.s and the shear viscosity $\eta_{s}$ to 500 Pa.s. Each particle has an internal length $R$ of $0.2 \mathrm{~m}$. We specify periodic boundary conditions along the vertical lines to model the infinite dimension in the shear direction. Along the horizontal lines the cosserat rotation and the normal velocity are set to zero and we specify a shear stress of $10^{4} \mathrm{~Pa}$ on top and $-10^{4} \mathrm{~Pa}$ at the bottom.

Initially (Fig. 4h), a random director orientation between $-\frac{\pi}{2}$ and $\frac{\pi}{2}$ is set to each particle. Thus the initial behaviour is isotropic. Particles along the central vertical line are "dyed" to track the material motion through time (Fig. (4). To each configuration (a), (b) and (c) corresponds a plot (a'), (b') and (c') of the isovalues of $n_{x}$. On the first profile (Fig. $4 \mathrm{a}^{\prime}$ ), we get a unique isovalue 0.64 which corresponds to average value of the cosinus of the orientation angle of the director.

While the shear stress is applied, grains are reorientated parallely to the shear direction (Fig. $\left.4 \mathrm{~b}^{\prime}\right)$. Once they reach the weakest orientation $\left(n_{x}=0\right)$, they remain in that position. As shown on plot 4 ', the material is now strongly anisotropic due to the preferential orientation of interpolation points.

\section{Conclusions}

The Cosserat model shows the internal length effect on flow velocity. The Cosserat theory coupled with the director theory in the shear model can explain qualitatively the anisotropy induced during strong shearing, for example in a silo discharge of non-spherical grains. 


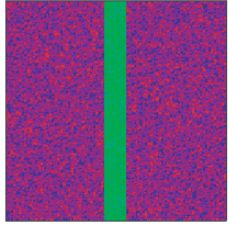

(a)

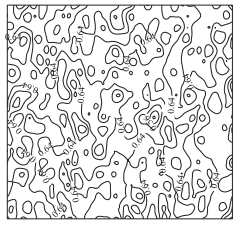

$\left(a^{\prime}\right)$

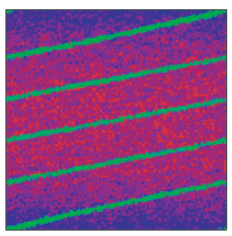

(b)

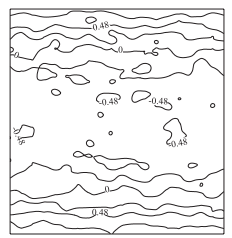

(b')

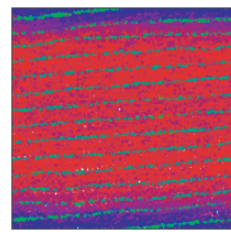

(c)

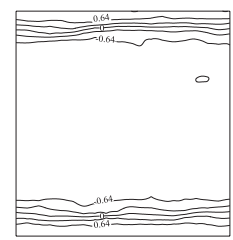

$\left(c^{\prime}\right)$

Fig. 4. (a) Initial conditions with random particle orientation, (b) beginning of the anisotropic behaviour and (c) the material is oriented. Plots (a'), (b') and (c') are the isovalues of the director orientation respectively corresponding to configuration (a), (b) and (c)

\section{References}

1. L. Moresi, F. Dufour, and H.-B. Mühlhaus. A lagrangian integration point finite element method for large deformation modeling of viscoelastic geomaterials. Journal of Computational Physics, In press, 2002.

2. H. Sakaguchi and H.-B. Mühlhaus. Mesh free modelling of failure and localization in brittle materials. In Deformation and Progressive Failure in Geomechanics, pages 15-21. Pergamon, 1997.

3. E. Cosserat and F. Cosserat. Théorie des corps déformables. Hermann, Paris, 1909.

4. H.-B. Mühlhaus, R. de Borst, and E.C. Aifantis. Constitutive models and numerical analysis for inelastic materials with microstrucutre. In Booker Eds. Beer and Carter, editors, Computer Methods and Advances in Geomechanics, pages 377385, Rotterdam, 1991. Balkema.

5. D.P. Adhikary, H.-B. Mühlhaus, and A.V. Dyskin. Modeling the large deformations in stratified media-the cosserat continuum approach. Mechanics of CohesiveFrictional Materials, 4:195-213, 1999.

6. S. Forrest, G. Cailletaud, and R. Sievert. A cosserat theory for elastoviscoplastic single crytals at finite deformation. Archives of Mechanics, 49(4):705-736, 1997.

7. L. Moresi, H.-B. Mühlhaus, and F. Dufour. Particle-in-cell solutions for creeping viscous flows with internal interfaces. In H.-B. Mühlhaus, A. Dyskin, and E. Pasternak, editors, In Bifurcation and Localization in Soils and Rocks, pages 345-353, Rotterdam, 2001. Balkema.

8. L. Moresi, H.-B. Mühlhaus, and F. Dufour. Viscoelastic formulation for modelling of plate tectonics. In H.-B. Mühlhaus, A. Dyskin, and E. Pasternak, editors, In Bifurcation and Localization in Soils and Rocks, pages 337-343, Rotterdam, 2001. Balkema. 
9. J.J. Monaghan. Smoothed particle hydrodynamics. Annual Review of Astronomy and Astrophysics, 30:543-574, 1992.

10. D. Sulsky, Z. Chen, and H.L. Schreyer. A particle method for history-dependent materials. Computer Methods in Applied Mechanics and Engineering, 118:179-196, 1994.

11. S.K. Choi and H.-B. Mühlhaus. Distinct elements vs Cosserat theory: A comparison for the case of an infinite shearlayer, pages 315-319. Eds. Beer, Booker and Carter, 1991. 